\title{
El Bullying en el País Vasco: Prevalencia y Diferencias en Función del Sexo y la Orientación-Sexual
}

\author{
Enara Larrain ${ }^{\mathrm{a}} \mathrm{y}$ Maite Garaigordobil ${ }^{\mathrm{b}}$
}

aTolosako Inmakulada Lanbide Ikastola, Guipúzkoa, España; 'bUniversidad del País Vasco, España

\section{INFORMACIÓN \\ DEL ARTÍCULO}

\section{Historia del artículo:}

Recibido el 9 de abril de 2020

Aceptado el 26 de mayo de 2020

Online el 24 de julio de 2020

\section{Palabras clave:}

Bullying

LGB

Discriminación

Acoso escolar

Adolescencia

Orientación sexual

\section{Keywords:}

Bullying

LGB

Discrimination

School harassment

Adolescence

Sexual orientation

\section{R E S U M E N}

Todos los estudiantes sufren el riesgo de ser víctima de conductas agresivas de bullying durante el transcurso de su vida. Sin embargo, existen colectivos con mayor riesgo de sufrir estas conductas, en especial, el colectivo LGTB. Este estudio tiene tres objetivos: (1) analizar la prevalencia de víctimas y agresores de bullying entre adolescentes de secundaria, (2) explorar diferencias en función del sexo y (3) conocer las diferencias en función de la orientación sexual. Una muestra compuesta por 1,748 adolescentes del País Vasco cumplimentó dos cuestionarios. Los resultados evidencian (1) un elevado porcentaje de víctimas ( $41.6 \%$ globales, $11 \%$ severas) y de agresores ( $28.5 \%$ globales, $2,7 \%$ severos), (2) que las chicas muestran mayor victimización y los chicos agresión y (3) que hay un mayor porcentaje de víctimas no-heterosexuales, en especial de gais y bisexuales. En conclusión, las personas LGTB muestran una mayor vulnerabilidad a sufrir acoso escolar, por lo que es necesario desarrollar e implementar programas antidiscriminatorios en la comunidad educativa.

\section{Bullying in the Basque Country: Prevalence and differences depending on sex and sexual orientation}

\section{A B S T R A C T}

Every student is at risk of aggressive bullying behavior during their lifetime. However, there are groups with a higher risk of suffering these aggressive behaviors, especially the LGTB group. This study has three objectives: (1) to analyze the prevalence of victims and aggressors of bullying among high school adolescents; (2) to explore differences based on sex; and (3) to identify differences based on sexual orientation. A sample consisting of 1,748 adolescents from the Basque Country completed two questionnaires. The results show (1) a high percentage of victims (41.6\% global, $11 \%$ severe), as well as aggressors (28.5\% global, $2.7 \%$ severe); (2) that girls show greater victimization and boys show aggression; and (3) a higher percentage of non-heterosexual victims, especially gay and bisexual. In conclusion, LGTB people show greater vulnerability to suffer bullying and, therefore, the need to develop and implement antidiscrimination programs in the educational community.
La definición más aceptada y utilizada de bullying (también denominado acoso escolar, maltrato entre iguales, violencia entre iguales, etc.) es la formulada por Olweus (1999). Este investigador considera que un estudiante está siendo intimidado cuando otro estudiante o grupo de estudiantes le dice cosas mezquinas o desagradables, se ríe de él o ella o le llama por nombres molestos o hirientes; le ignora completamente, le excluye de su grupo de amigos o amigas o le retira de actividades a propósito; le golpea, patea y empuja o le amenaza; cuenta mentiras o falsos rumores sobre él o ella, le envía notas hirientes y trata de convencer a los demás para que no se relacionen con él o ella, etc. Estas conductas ocurren frecuentemente y es difícil para el estudiante que está siendo intimidado defenderse por sí mismo. Tam- bién es bullying cuando a un estudiante le molestan repetidamente de forma negativa y dañina. En resumen, el acoso escolar se caracteriza por la intención de hacer daño repetidamente en una situación en la que existe un desequilibrio de poder entre la víctima y el agresor (Olweus, 2013).

Los estudios que han analizado este fenómeno permiten distinguir cuatro formas de conductas agresivas de bullying cara a cara: (1) física, tales como conductas agresivas directas dirigidas contra el cuerpo, como pegar o empujar, o conductas agresivas indirectas dirigidas contra la propiedad (robar, romper, ensuciar, esconder objetos, etc.); (2) verbal, como conductas verbales negativas (insultos, motes, hablar mal de esa persona, calumnias...); (3) social, referida a conductas

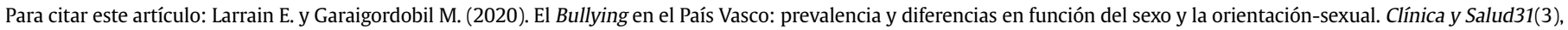
147-153. https://doi.org/10.5093/clysa2020a19

Financiación. El estudio forma parte de un trabajo más amplio financiado por el Dpto. de Educación, de Gobierno Vasco (PRE_2017_1_0017) y se encuadra en el marco de las acti-

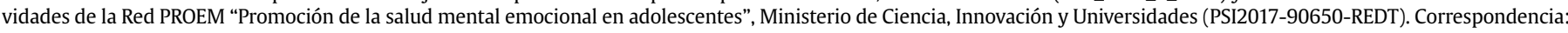
enaralarrain@gmail.com (E. Larrain).

ISSN:1130-5274/@ 2020 Colegio Oficial de la Psicología de Madrid. This is an open access article under the CC BY-NC-ND license (http://creativecommons.org/licenses/by-nc-nd/4.0/). 
mediante las cuales se aísla al individuo del grupo (no se le deja participar en alguna actividad, se le margina, aísla, ignora...); (4) psicológica, que aluda a las formas de acoso que afectan a la autoestima y crean inseguridad y miedo (se ríen de la víctima, la desvalorizan, la humillan, la acechan creándole sentimientos de indefensión y temor, etc.) (Garaigordobil, 2013; Garaigordobil y Oñederra, 2010).

Los estudios de prevalencia dirigidos a analizar los porcentajes de víctimas y agresores en adolescentes de educación secundaria de escuelas del País Vasco (Garaigordobil, 2013; Garaigordobil, 2015a, 2015b) han mostrado una prevalencia de bullying global (una o más veces) de $39.2 \%$ víctimas y $38.4 \%$ agresores. Entre las conductas de bullying destacan en primer lugar las verbales (34.4\%), seguidas de las sociales (10.6\%), físicas (9.5\%) y psicológicas (9.1\%). Respecto al bullying severo (bastantes veces y siempre), una revisión reciente (ver revisión Garaigordobil, 2017) evidenció un porcentaje de entre $3.8 \%$ y $14.3 \%$ en víctimas y entre un $2.4 \%$ y un $9.8 \%$ de agresores en adolescentes de segundo ciclo de secundaria que participaron en distintos estudios españoles. En cuanto al porcentaje de implicados en bullying cara a cara en el resto del mundo, Garaigordobil (2017) mostró en la revisión realizada un porcentaje de entre $2.7 \%$ y $27.9 \%$ de víctimas severas y entre un $6 \%$ y un $23.1 \%$ de agresores severos. Por lo tanto, aunque se hayan puesto en marcha distintos programas de prevención y de intervención, los porcentajes de victimización y agresión siguen siendo alarmantes. En relación a las diferencias en función del sexo, estos estudios han evidenciado que el porcentaje de varones y mujeres víctimas fue similar, aunque se ha observado un mayor porcentaje de agresores varones.

El bullying es un fenómeno que afecta a toda la comunidad educativa. No obstante, diversos autores concluyen que el colectivo LGB (lesbianas, gais y bisexuales) es un colectivo más vulnerable a padecer victimización en comparación con las personas heterosexuales (Abreu y Kennym, 2017; Baiocco et al., 2018; Camodeca et al., 2018; COGAM, 2016; Elipe et al., 2017; Gegenfurtner y Gebhardt, 2017; Toomey y Russel, 2016). Del mismo modo, las personas que no tienen clara su orientación afectivo-sexual (Birkett et al., 2009) también muestran mayores niveles de victimización en comparación con sus iguales. Respecto a la prevalencia, distintas investigaciones han arrojado datos que dan porcentajes entre 51\% y 57.38\% de victimización de acoso entre las personas con una orientación sexual no-normativa (Generelo et al., 2012; Martxueta y Etxeberria, 2014).

Otras investigaciones se han centrado en comparar la prevalencia de acoso escolar dentro del colectivo LGB. Pichardo et al. (2013) encontraron que las personas homosexuales mostraban mayor prevalencia de victimización en comparación con las personas bisexuales (51.27\% vs 42.84\%). Así mismo, Orue et al. (2018) en una investigación reciente han encontrado que en las personas homosexuales los chicos gais son los que mayor victimización sufren en comparación con las chicas lesbianas. Finalmente, con el objetivo de profundizar más en estas comparaciones, Abreu y Kennyim (2017) han podido observar que mientras que en las chicas las bisexuales son las que mayor índice de victimización muestran en los chicos el colectivo más vulnerable es el de los homosexuales. Este último dato fue previamente sacado a la luz por COGAM (2016).

Aunque haya investigaciones que han analizado la prevalencia del bullying cara a cara en los adolescentes del País Vasco, pocos de estos estudios no analizan las diferencias en función de la orientación sexual.

\section{Objetivos e Hipótesis}

El estudio se plantea 3 objetivos, que incluyen 6 hipótesis:

Objetivo 1: analizar la prevalencia de bullying cara-a-cara entre los adolescentes de segundo ciclo de secundaria del País Vasco. En base a la revisión realizada de estudios previos, se plantean 3 hipótesis:
H1. El porcentaje de víctimas de bullying global (han sufrido estas conductas algunas veces, bastantes veces y siempre) en estudiantes de segundo ciclo de secundaria del País Vasco será aproximadamente de $30 \%$, de los cuales aproximadamente un 10\% habrá sufrido estas conductas de forma frecuente, es decir, bastantes veces y siempre (víctimas severas).

$H 2$. El porcentaje de agresores de bullying global en estudiantes de segundo ciclo de secundaria del País Vasco será aproximadamente de un $30 \%$, de los cuales aproximadamente un $6 \%$ serán severos, es decir, se comportan así de forma muy frecuente.

$H 3$. Las conductas más frecuentes informadas por víctimas y agresores severos serán las conductas agresivas verbales, seguidas por las sociales, psicológicas y físicas.

Objetivo 2: explorar diferencias en función del sexo. Teniendo en cuenta los estudios previos, se plantea una hipótesis:

H4. Se encontrará mayor porcentaje de víctimas chicas y mayor porcentaje de agresores chicos. Las chicas sufrirán más conductas agresivas (nivel de victimización), mientras que los chicos perpetrarán en mayor grado estas conductas (nivel de agresión).

Objetivo 3: comparar el porcentaje de víctimas y agresores y el grado de conductas agresivas sufridas y perpetradas en personas heterosexuales, gais, lesbianas, bisexuales y las que no están seguras de su orientación sexual. Con este objetivo, se plantean dos hipótesis:

H5. Se hallará mayor porcentaje de víctimas de bullying no heterosexuales comparadas con las heterosexuales. Dentro del grupo no heterosexual, habrá un mayor porcentaje de víctimas en el colectivo de gais y bisexuales. Además, los bisexuales será el grupo que sufrirá más conductas agresivas (nivel de victimización).

H6. No se hallarán diferencias entre heterosexuales y no heterosexuales agresores, aunque en los no heterosexuales habrá un mayor porcentaje de agresoras lesbianas. Además, las lesbianas será el grupo que más conducta agresiva perpetre (nivel de agresión).

\section{Método}

\section{Participantes}

La muestra del estudio está constituida por 1,748 adolescentes de

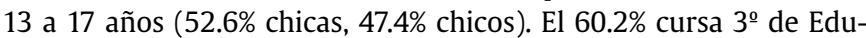
cación Secundaria y $39.8 \%$ estudia $4^{0}$ curso. El $44.7 \%$ están inscritos en centros públicos y el $55.3 \%$ en privados. Respecto a la orientación sexual, el $87.5 \%$ son heterosexuales, $5.9 \%$ no estaban seguros/as de su orientación sexual, $5.7 \%$ son bisexuales, $0.7 \%$ gais y $0.2 \%$ lesbianas. Es decir, el $12.5 \%$ son no heterosexuales y $87.5 \%$ lo son. La muestra fue seleccionada aleatoriamente, siendo representativa de estudiantes del último ciclo de secundaria del País Vasco $(N=37.575)$. Utilizando un nivel de confianza de $95 \%$, con un error muestral de $2.3 \%$, la muestra representativa es de 1,732 . Para la selección de la muestra se utilizó una técnica de muestreo estratificado, teniendo en cuenta los siguientes parámetros: provincia, tipo de red (público, privado) y nivel educativo ( $3^{\circ}$ y $4^{\circ}$ cursos).

\section{Procedimiento}

El estudio utiliza una metodología descriptiva y comparativa, de corte transversal. En primer lugar, se envió una carta a los centros educativos seleccionados aleatoriamente explicando la investigación. Aquellos que aceptaron participar recibieron el consentimiento informado para padres y participantes. Posteriormente, el equipo evaluador se desplazó a los centros y administró a los estudiantes los instrumentos de evaluación. El estudio cumplió los valores éticos 
requeridos en la investigación con seres humanos, habiendo sido evaluado favorablemente por la Comisión de Ética de la UPV/EHU (M10_2017_094).

\section{Instrumentos}

Para medir las variables objeto de estudio, en primer lugar se utilizó un cuestionario sociodemográfico (Garaigordobil y Larrain, 2017) que recoge información de variables como edad, curso, sexo, orientación sexual, identidad sexual, etc. En segundo lugar, para medir el bullying se utilizó un instrumento con garantías psicométricas de fiabilidad y validez, Cyberbullying: Screening de Acoso entre Iguales (Garaigordobil, 2013, 2017). La prueba evalúa bullying presencial y cyberbullying. La escala de bullying mide cuatro tipos de acoso presencial o "cara a cara" (físico, verbal, social y psicológico) y la escala de cyberbullying explora 15 conductas relacionadas con el acoso cibernético (enviar mensajes ofensivos/amenazadores, telefonear para insultar/amenazar, grabar una agresión/humillación y difundir el vídeo, crear rumores para difamar, robar la contraseña, aislar en redes sociales, etc.). Los adolescentes informan de la frecuencia con la que han sufrido o perpetrado estas conductas en el transcurso de su vida. El test permite distinguir entre víctimas/agresores globales y severas. Las víctimas/agresores globales son aquellos participantes que han sufrido o perpetrado conductas agresivas de bullying al menos una vez durante el transcurso de la vida. Las víctimas/agresores severos en cambio, se refieren a aquellos participantes que han sufrido o realizado bullying cara a cara frecuentemente, es decir, bastantes veces o siempre.

Además, se obtiene una puntuación del nivel de victimización, cibervictimización, agresión y ciberagresión, es decir, el grado en que han sufrido o perpetrado conductas sufridas o perpetradas de modo presencial o tecnológicamente. En este estudio se utiliza únicamente la escala de bullying. Los coeficientes alfa de Cronbach de la muestra original evidencian una consistencia interna adecuada (bullying, $\alpha=.81$, cyberbullying, $\alpha=.91$ ), en la misma dirección que la muestra de este estudio (bullying, $\alpha=.76$, cyberbullying, $\alpha=.84$ ).

\section{Análisis de Datos}

Para conocer la prevalencia de estudiantes víctimas y agresores se calculan la frecuencia y el porcentaje de estudiantes que ha sufrido y perpetrado conductas de bullying globalmente (algunas veces, bastantes veces o siempre) o gravemente de modo frecuente (bastantes veces o siempre). Con el objetivo de proporcionar datos más específicos sobre las conductas agresivas de bullying, se calculan frecuencias y porcentajes en función del tipo de agresión y de los dos roles evaluados (víctima y agresor) de manera severa. Para identificar si existen diferencias en función del sexo en los dos indicadores de bullying (victimización y agresión), se llevan a cabo análisis descriptivos (media y desviación típica), análisis de varianza univariante y tamaño del efecto (prueba $d$ de Cohen), con las puntuaciones de chicas y chicos.

Además, con el objetivo de analizar si existen diferencias en la prevalencia de bullying global en función de la orientación sexual, se calculan frecuencias, porcentajes, chi cuadrado de Pearson y V de Cramer, teniendo en cuenta los participantes heterosexuales, gais, lesbianas, bisexuales y aquellos que no estaban seguros de su orientación sexual. Complementariamente, para verificar si existen diferencias entre los cinco grupos de orientación sexual en los dos indicadores de bullying (victimización y agresión), se llevan a cabo análisis descriptivos (media y desviación típica), análisis de varianza univariantes y tamaño del efecto (eta cuadrado), así como análisis post hoc de Bonferroni de comparación de grupos.

\section{Resultados}

\section{Víctimas y Agresores de Bullying: Prevalencia Global, Severa y Conductas más Frecuentes}

En relación a la prevalencia de bullying global se produjeron los siguientes resultados: (1) víctimas globales: el 41.6\% de los participantes ha sufrido conductas agresivas cara a cara alguna vez en el transcurso de su vida y (2) agresores globales: el 28.5\% de los participantes ha perpetrado conductas agresivas cara a cara alguna vez en la vida (ver Tabla 1 ).

Respecto a la prevalencia del bullying severo, es decir, en relación al porcentaje de estudiantes que ha sufrido frecuentemente conductas agresivas los resultados fueron: (1) víctimas severas: el 11\% de los participantes ha sufrido conductas agresivas de bullying cara a cara frecuentemente en el transcurso de su vida y (2) agresores severos: el $2.7 \%$ de los participantes ha perpetrado conductas agresivas de bullying cara a cara frecuentemente en la vida (ver Tabla 1).

De las diferentes conductas agresivas exploradas, los participantes indican que el tipo de agresión más frecuente que sufren los estudiantes son las agresiones verbales (8.8\%), seguidas de las sociales (4.3\%), psicológicas (4\%) y físicas (1.6\%).

En síntesis, el porcentaje de víctimas globales fue de $41.6 \%$, de los cuales el $11 \%$ eran víctimas severas y sufrían bullying frecuentemente, mientras que el porcentaje de agresores globales fue de $28.5 \%$, de los cuales $2.7 \%$ eran agresores severos y perpetraban estas conductas muy frecuentemente. Las conductas agresivas más frecuentes son las verbales, seguidas de las de exclusión social.

\section{Víctimas y Agresores de Bullying: Diferencias en Función del Sexo}

El porcentaje de mujeres y varones víctimas severas sobre la muestra en cada sexo fue de $9.5 \%(n=131)$ de mujeres y $4.6 \%(n=$ $62)$ de varones. Se hallaron diferencias estadísticamente significativas en función del sexo $\left(\chi^{2}=15.54, p<.001\right)$, con mayor porcentaje de víctimas mujeres. En cuanto a las diferencias en función del sexo en agresores severos, el porcentaje de mujeres y varones agresores severos sobre la muestra en cada sexo fue de $2.6 \%$ mujeres $(n=23)$ y $2.8 \%$ varones $(n=24)$, no hallándose diferencias estadísticamente significativas entre ambos $\operatorname{sexos}\left(\chi^{2}=0.04, \mathrm{~V}=.01, p>.05\right)$.

En relación al grado en que se ha sufrido o perpetrado la conducta agresiva, los resultados de los análisis de varianza muestran diferencias estadísticamente significativas en función del sexo en los indicadores de victimización y agresión (ver Tabla 1). Las chicas tienen significativamente puntuaciones medias más altas en el nivel de victimización y los chicos en el nivel de agresión. Por lo tanto, las chicas sufren más conductas agresivas y los chicos perpetran más conductas de este tipo, aunque el tamaño del efecto de estas diferencias es pequeño.

En síntesis, se encontró mayor porcentaje de víctimas mujeres pero un porcentaje similar de agresores y agresoras. Además, las chicas sufren más conductas agresivas y los chicos perpetran más este tipo de conductas.

Tabla 1. Víctimas y agresores: frecuencias y porcentajes de participantes en función de la frecuencia

\begin{tabular}{lcc}
\hline & Globales & Severos \\
\cline { 2 - 3 } & $f(\%)$ & $f(\%)$ \\
\hline Víctimas & $728(41.6)$ & $193(11)$ \\
Agresores & $500(28.5)$ & $47(2.7)$ \\
\hline
\end{tabular}

\section{Víctimas y Agresores de Bullying. Diferencias en Función de la Orientación Sexual}

Del $11 \%$ de las víctimas severas $(n=193)$ el porcentaje de heterosexuales y no heterosexuales sobre la muestra en cada grupo de 
orientación sexual fue de $9 \%$ heterosexuales $(n=138)$ y $25.1 \%$ no heterosexuales $(n=55)$, hallándose diferencias significativas $\left(\chi^{2}=50.48\right.$, $\mathrm{V}=.51, p<.001)$ con mayor porcentaje de víctimas en el grupo no heterosexual. El porcentaje de víctimas en cada grupo de orientación sexual en función de la muestra en cada grupo fue de $76.9 \%$ gais $(n=$ 10), $75.8 \%$ bisexuales $(n=75), 60.6 \%$ no están seguros de su orientación sexual $(n=63), 37.9 \%$ heterosexuales $(n=579)$ y $33.3 \%$ lesbianas $(n=2)$. Se hallaron diferencias estadísticamente significativas en función de la orientación sexual $\left(\chi^{2}=78.46, \mathrm{~V}=.63, p<.001\right)$, con mayor porcentaje de víctimas gais y bisexuales.

Del 2.7\% $(n=47)$ de los agresores severos el porcentaje de heterosexuales y no heterosexuales sobre la muestra en cada grupo de orientación sexual fue $1.7 \%$ heterosexuales $(n=38)$ y $0.9 \%$ no heterosexuales $(n=9)$, no hallándose diferencias significativas en función de la orientación sexual $\left(\chi^{2}=0.75, \mathrm{~V}=.12, p>.05\right)$. El porcentaje de agresores en cada grupo de orientación sexual en función de la muestra en cada grupo fue de $27.4 \%$ heterosexuales $(n=419), 38.5 \%$ gais $(n=5)$, $66.7 \%$ lesbianas $(n=2), 45.5 \%$ bisexuales $(n=54)$ y $27.9 \%$ participantes que no están seguros de su orientación sexual $(n=29)$. Se hallaron diferencias estadísticamente significativas en función de la orientación sexual $\left(\chi^{2}=17.61, \mathrm{~V}=.61, p<.05\right)$, con mayor porcentaje de agresores en el grupo de lesbianas.

En relación al grado en que se sufrió o perpetró la conducta en función de la orientación sexual, los resultados se presentan en la Tabla 2. Los resultados evidencian que las personas bisexuales son las que tienen puntuaciones más altas en victimización. En relación al nivel de agresión, se evidencian puntuaciones medias significativamente más altas en el grupo de las lesbianas. Por consiguiente, los bisexuales fueron los que habían sufrido significativamente más conductas agresivas de bullying cara a cara en el transcurso de la vida y las lesbianas fueron las que habían perpetrado significativamente más conductas de este tipo.

En síntesis, hay un mayor porcentaje de víctimas severas no heterosexuales, en las cuales se evidencia un mayor porcentaje de víctimas en gais y bisexuales. No se hallan diferencias en agresores severos en función de la orientación sexual, aunque se obtiene un mayor porcentaje de agresoras lesbianas. Además, las personas bisexuales son las sufren más conductas agresivas (nivel de victimización) y las lesbianas las que perpetran más conductas agresivas (nivel de agresión).

Tabla 2. Medias, desviaciones típicas, análisis de varianza y tamaño del efecto ( $d$ de Cohen) en indicadores de bullying en mujeres y varones

\begin{tabular}{lccrc}
\hline & $\begin{array}{c}\text { Mujeres } \\
(n=920)\end{array}$ & $\begin{array}{c}\text { Varones } \\
(n=828)\end{array}$ & $F(p)$ & $d$ \\
\cline { 2 - 5 } & $M(D T)$ & $M(D T)$ & & \\
\hline Victimización & $0.78(1.84)$ & $1.17(1.44)$ & $23.00(.000)$ & 0.23 \\
Agresión & $0.42(0.92)$ & $0.52(0.93)$ & $5.04(.025)$ & 0.10 \\
\hline
\end{tabular}

\section{Discusión}

Este estudio tuvo como objetivos: (1) analizar la prevalencia de bullying cara a cara en los adolescentes de segundo ciclo de secundaria del País Vasco, (2) explorar diferencias en función del sexo y (3) comparar el porcentaje de víctimas y agresores y el grado en que se sufre o perpetran estas conductas en personas heterosexuales, gais, lesbianas, bisexuales y aquellas que no están seguras de su orientación sexual.

En primer lugar, se evidencia un $41.6 \%$ de víctimas de bullying cara a cara global y un $11 \%$ de víctimas severas. De esta manera, la hipótesis 1 se cumple en lo que respecta al rol de víctima severa, puesto que el porcentaje de víctimas globales ha sido incluso superior al esperado. Estos resultados ratifican lo hallado en una revisión reciente en la que se pone de manifiesto que en algunas investigaciones el porcentaje de estudiantes que sufre conductas agresivas presenciales de una manera ocasional supera el 80\% (Bogolyubova et al., 2015; ver revisión de Garaigordobil, 2017). Además, cabe destacar que en una investigación en la que se evaluó la prevalencia de bullying en adolescentes de 12-18 años del País Vasco (Garaigordobil, 2015b) se obtuvo un porcentaje de $39.2 \%$ de víctimas. Así se confirma que el bullying es un fenómeno que continúa con notable crecimiento, por lo que es necesario seguir trabajando en su investigación y en el desarrollo de actividades y programas de prevención y de intervención.

En segundo lugar, en cuanto al rol de agresor se evidencia un $28.5 \%$ de agresores globales, de los cuales un $2.7 \%$ son agresores severos. Así, la hipótesis 2 se cumple parcialmente, habiendo encontrado un porcentaje similar al esperado de agresores globales y un porcentaje inferior de agresores severos. Hay que tener en cuenta que los datos epidemiológicos que aparecen en esta investigación son fruto de autoinformes cumplimentados por los propios alumnos, con los sesgos de deseabilidad social que implican. Esto nos lleva a pensar que el porcentaje de agresores en realidad puede ser algo superior al informado por los estudiantes, lo cual confirmaría los resultados de la revisión de Garaigordobil (2017), que encuentra estudios en los que hasta un $45 \%$ de agresores han perpetrado conductas agresivas de bullying una o más veces durante el transcurso de la vida.

Como se puede observar, los porcentajes de estudiantes que ha sufrido bullying cara a cara de manera ocasional y frecuentemente son para tener en cuenta. Estos datos evidencian que aunque durante los últimos años se haya realizado un gran esfuerzo en el diseño e implementación de programas de prevención e intervención de bullying, y aunque distintas entidades públicas hayan trabajado con el objetivo de frenar este fenómeno, el bullying presencial continúa siendo una problemática que está presente en nuestra sociedad.

En relación a las conductas de bullying presencial más frecuentes de las que dan cuenta los estudiantes, los resultados muestran que las conductas agresivas verbales son las más frecuentes, seguidas por las sociales, psicológicas y físicas. Por ello, la hipótesis 3 se cumple en su totalidad. Estos resultados apuntan en la misma línea que los encontrados en la revisión realizada por Garaigordobil (2017), ya que, al parecer, a medida que aumenta la edad de los estudiantes las conductas físicas disminuyen, mientras que las sociales y psicológicas aumentan.

Respecto a las diferencias en función del sexo en el porcentaje de víctimas y agresores de bullying presencial severo, se evidencia mayor porcentaje de víctimas chicas y un porcentaje similar de agresores en ambos sexos. Sin embargo, se han encontrado diferencias en el nivel de agresión y victimización en función del sexo. Mientras que las chicas sufren mayor cantidad de conductas agresiva, los chicos perpetran más conductas de bullying presencial. Por lo tanto, aunque no se hayan encontrado diferencias en los porcentajes de agresores y agresoras, la hipótesis 4 se cumple casi en su totalidad. Los resultados hallados en esta investigación confirman lo encontrado en estudios previos (Garaigordobil, 2017), en los que se pone de relieve que los chicos tienden a perpetrar conductas agresivas con mayor frecuencia. No obstante, cabría destacar que en las chicas la presión social les lleva a mostrarse ante la sociedad como el sexo no agresivo, atento, sensible, etc. y en el caso de los chicos, en cambio, se muestran como el sexo fuerte, agresivo, provocador.

En cuanto a las diferencias en el porcentaje de víctimas en función de la orientación sexual, este estudio evidencia un mayor porcentaje de víctimas de bullying en personas no heterosexuales en comparación con las heterosexuales. Además, en los chicos gais y las personas bisexuales hay mayor porcentaje de víctimas. Finalmente, el colectivo de bisexuales sufre más conductas agresivas en comparación con los demás grupos no heteronormativos. De este modo, la hipótesis 5 se cumple en su totalidad. Estos resultados apuntan en dirección similar a la señalada por otros estudios (Abreu y Kennym, 2017; Baiocco et al., 2018; Camodeca et al., 2018; COGAM, 2016; Elipe et al., 2017; Gegenfurtner y Gebhardt, 2017; Orue et al., 2018; Toomey y Russel, 2016), en los que se evidencia que el colectivo de personas no hetero 


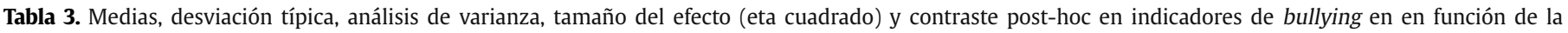
orientación sexual

\begin{tabular}{|c|c|c|c|c|c|c|c|c|c|}
\hline & $\begin{array}{c}\text { Heterosexual } \\
(n=1,529)\end{array}$ & $\begin{array}{c}\text { Gay } \\
(n=13)\end{array}$ & $\begin{array}{l}\text { Lesbiana } \\
(n=3)\end{array}$ & $\begin{array}{l}\text { Bisexual } \\
(n=99)\end{array}$ & $\begin{array}{c}\text { No } \\
\text { seguro/a } \\
(n=104) \\
\end{array}$ & $F$ & $p$ & $\eta^{2}$ & Post hoc \\
\hline & $M(D T)$ & $M(D T)$ & $M(D T)$ & $M(D T)$ & $M(D T)$ & & & & \\
\hline Victimización & $0.84(1.53)$ & $1.69(1.37)$ & $1.67(2.88)$ & $2.58(2.48)$ & $1.42(1.79)$ & 29.23 & .000 & .063 & $1=2=3 ; 1<4.5 ; 2=3=4=5 ; 4>5$ \\
\hline Agresión & $0.44(0.92)$ & $0.69(1.18)$ & $2.33(2.51)$ & $0.74(1.03)$ & $0.40(0.74)$ & 5.66 & .000 & .013 & $1=2=5 ; 1<3.4 ; 2=3.4 ; 3>4.5$ \\
\hline
\end{tabular}

Nota. 1 = heterosexual; 2 = gay; 3 = lesbiana; 4 = bisexual; 5 = no seguro/a.

normativas son un colectivo más vulnerable en comparación con las personas que se encuentran dentro de los cánones de la heteronormatividad.

Otro de los puntos importantes en este sentido es que en este trabajo las personas bisexuales muestran un porcentaje elevado de victimización cuando se analiza el bullying en función de la orientación sexual (Tabla 3). Estos resultados contrastan con lo encontrado por Pichardo et al. (2013) en una investigación realizada a nivel estatal, en la que halló que las personas homosexuales sufren mayor victimización que las bisexuales. En este sentido, hay que tener en cuenta que conseguir una muestra de personas con una orientación sexual no normativa es un trabajo complicado. Aunque vivamos en una sociedad en la que se ha avanzado mucho en lo que se refiere a la diversidad sexual, aún existe una gran estigmatización y un gran miedo a ser una persona que se aleja de los estereotipos impuestos por la sociedad. En muchos casos, para un adolescente es más fácil admitir que es bisexual a decir que es homosexual, ya que las personas bisexuales podrán seguir teniendo en algunos aspectos los "privilegios" que tienen las personas heterosexuales. De ese modo, se puede suponer que en esta muestra habrá un número de adolescentes homosexuales que quizás se hayan podido identificar como bisexuales.

Respecto a las diferencias en el porcentaje de agresores severos de bullying presencial en función de la orientación sexual, no se han encontrado diferencias entre heterosexuales y no heterosexuales, aunque se constata un mayor porcentaje de agresores en el colectivo de lesbianas. Complementariamente, las lesbianas perpetran significativamente más conductas agresivas (nivel de agresión) comparadas con los otros grupos de orientación sexual. Es decir, la hipótesis 6 se cumple en su totalidad. Uno de los aspectos que cabe tener en cuenta es que el número de chicas lesbianas de este estudio es muy escaso, hecho que ha podido influir en los resultados. En esta línea, teniendo en cuenta que el colectivo LGB es un colectivo más vulnerable a sufrir cualquier tipo de conducta agresiva, discriminación o rechazo, se puede suponer que, al menos en parte, las conductas agresivas que perpetran las personas LGB lo son en defensa propia o para demostrar fortaleza ante las personas que podrían ser sus agresores.

En conclusión, los datos evidencian un porcentaje considerable de víctimas y agresores de bullying cara a cara global y severo entre los estudiantes de segundo ciclo de secundaria del País Vasco. Además, la comparación con estudios previos llevados a cabo con la misma población confirma que es un fenómeno que, lejos de haber sido erradicado mediante los distintos programas y actividades antibullying, no solo sigue presente en los adolescentes de nuestra sociedad, sino que continúa aumentando en las aulas de nuestros centros educativos. En relación a los resultados en torno a la diversidad sexual, se evidencia que los adolescentes con una orientación afectivo-sexual no normativa son un colectivo de mayor vulnerabilidad a padecer conductas agresivas de bullying en su día a día, siendo un colectivo que sufre rechazo y discriminación por sus iguales tan solo por mostrarse fuera de los cánones que marca nuestra sociedad.

Estos resultados confirman una vez más que, aunque existan programas antibullying y políticas antidiscriminatorias que quieran fomentar el respeto por la diversidad sexual desde edades muy tempranas, la situación legal se encuentra muy lejos de la realidad social. Es decir, existen leyes que acusan las actitudes de rechazo y discriminación hacia el colectivo LGTB, aunque hasta el momento muchas personas continúan teniendo conductas y actitudes LGTB fóbicas. Desde la temprana infancia se continúa recibiendo mensajes meramente heteronormativos desde los medios de comunicación, entorno familiar, social y, sobre todo, desde la comunidad educativa (profesores, materiales audiovisuales, didácticos, etc.). Así mismo, estos resultados nos sitúan frente la obligación de continuar trabajando en el diseño de nuevas medidas para combatir todo tipo de agresión en los centros escolares, sin olvidar ningún tipo de origen que pueda tener cada una de ellas (orientación sexual, identidad sexual, raza, físico, etc.).

Este estudio muestra, algunas limitaciones. La mayor de ellas ha sido la obtención de la muestra ya que, por un lado, el bullying es un fenómeno que crea gran controversia y algunos de los centros escolares seleccionados aleatoriamente no accedieron a participar en esta investigación. Por otro lado, podría ser que los participantes no hayan sido totalmente sinceros respecto a su orientación sexual y haberse identificado como heterosexuales por miedo a ser rechazados entre sus compañeros. Por último, la utilización de cuestionarios de autoinforme trae como consecuencia el sesgo de deseabilidad social, que habría que tener en cuenta.

Estos resultados ratifican la necesidad de continuar desarrollando e implementando programas de prevención e intervención del acoso escolar. A este respecto, se podría mencionar el programa de intervención Cyberprogram 2.0 (Garaigordobil y Martínez-Valderrey, 2014, 2016, 2018), que ha conseguido reducir el bullying presencial y que además incluye algunas actividades sobre al acoso debido a la orientación sexual. En esta línea, es necesario diseñar nuevas actividades que fomenten el respeto hacia las personas LGTB y hacia aquellas personas que se alejen de los estereotipos que están marcados por la heteronormatividad. Estas actividades deberían estar dirigidas a toda la comunidad educativa, comenzando desde los estudiantes de más temprana edad, continuando con los estudiantes en etapas de la adolescencia y finalizando con actividades específicas para padres/ madres y todos los trabajadores de las escuelas. Es imposible conseguir un cambio en el alumnado si no se educa a los principales educadores de nuestra sociedad.

\section{Extended Summary}

Olweus (1999) was the author who defined the concept of bullying. This researcher considers that a person is being bullied when he or she is exposed, repeatedly and over time, to negative actions on the part of one or more persons, and he or she has difficulty defending himself or herself. The studies that have analysed this phenomenon allow us to distinguish 4 forms of aggressive behaviour: (1) physical, i.e., direct aggressive behaviour directed against the body or indirect aggressive behaviour directed against property; (2) verbal, i.e., verbal negative behaviours; (3) social, i.e., behaviours by which the individual is isolated; and (4) psychological, i.e., forms of harassment that corrode self-esteem and create insecurity and fear.

Prevalence studies aimed at analysing the percentages of victims and aggressors in adolescents from secondary education in schools in the Basque Country (Garaigordobil, 2013; Garaigordobil, 2015ab) have shown a prevalence of global bullying (one or more times) of 
$39.2 \%$ victims and $38.4 \%$ aggressors. Among bullying behaviors, verbal (34.4\%) stands out first, followed by social (10.6\%), physical (9.5\%), and psychological (9.1\%). Regarding severe bullying (quite a few times and always), a recent review (see review Garaigordobil, 2017) showed a percentage of between $3.8 \%$ and $14.3 \%$ victims and between $2.4 \%$ and a $9.8 \%$ aggressors among adolescents in the second cycle of secondary school who participated in different Spanish studies. In relation to the differences according to sex, these studies have shown that the percentage of male and female victims was similar, though a higher percentage of male aggressors was observed.

Bullying is a phenomenon that affects the entire educational community. However, various authors conclude that the LGB group and people who are not sure about their sexual orientation are more vulnerable to victimization compared to heterosexual people. Regarding prevalence, different studies yield percentages between $51 \%$ and $57.38 \%$ of victimization of bullying among people with non-normative sexual orientation.

This study had three objectives: (1) to analyse the prevalence of bullying among adolescents in the second cycle of secondary school in the Basque Country; (2) to explore differences based on sex; and (3) to compare the percentage of victims and aggressors and the amount of aggressive behaviours suffered and carried out, among heterosexual, gay, lesbian, bisexual people, and those who are not sure of their sexual orientation.

The sample is made up of 1,748 adolescents from 13 and 17 years old (52.6\% girls, $47.4 \%$ boys); $60.2 \%$ are in 3rd grade of Secondary Education and $39.8 \%$ are in 4 th grade; $44.7 \%$ are studying in public centres and $55.3 \%$ in private schools. Regarding sexual orientation, $87.5 \%$ are heterosexual, $0.7 \%$ gay, $0.2 \%$ lesbian, $5.7 \%$ bisexual, and $5.9 \%$ are not sure of their sexual orientation.

This study uses a descriptive and comparative, cross-sectional methodology. First, a letter was sent to randomly selected schools explaining the research. Those who agreed to participate received informed consents for parents and participants. Subsequently, the evaluation team travelled to the schools and administered the evaluation instruments to the students. The study had the ethical values required in research with human beings, having been favourably evaluated by the UPV/EHU Ethics Commission (M10_2017_094).

The research used two questionnaire to measure the variables: a socio-demographic questionnaire, which collects information on variables such us age, course, sex, sexual orientation, etc. and an instrument, with psychometric guarantees of reliability and validity, screening cyberbullying and harassment among equals. This test evaluates face-to-face bullying and cyberbullying. The bullying scale measures 4 types of face-to-face bullying (physical, verbal, social, and psychological. Adolescents report the frequency with which they have suffered and carried out these behaviours in the course of their lives. Cronbach's alpha coefficients with the original sample show adequate internal consistency (bullying $\alpha=.81$; cyberbullying $\alpha=$ .91 ), in the same direction as with the sample in this study (bullying $\alpha=.76$; cyberbullying $\alpha=.84$ ). Results were drawn after carrying out data analysis.

- Victims and aggressors of bullying: global prevalence, severe and more frequent behaviours.

- Global bullying. (1) Victims: 41.6\% of the participants have suffered aggressive face-to-face behaviours sometime in their lifetime. (2) Aggressors: $28.5 \%$ of participants have engaged in aggressive face-to-face behaviours at some time in their lives.

- Severe bullying. (1) Victims: 11\% of the participants have suffered aggressive behaviours frequently in their lifetime. (2) Aggressors: $2.7 \%$ of participants have engaged in aggressive face-to-face bullying behaviours frequently during their life.

- The most frequent type of aggression suffered by students is verbal aggression (8.8\%), followed by social (4.3\%), psychological (4\%) and the physical ones (1.6\%).
- Victims and aggressors of bullying: Differences according to sex.

- The percentage of women and men severely victims of the sample in each sex was $9.5 \%$ women $(n=131)$ and $4.6 \%$ men $(n=62)$. Statistically significant differences were found according to sex $\left(\chi^{2}=15.54, p<.001\right)$, with a higher percentage of female victims. Regarding the differences as a function of sex in severe aggressors, the percentage of women and men severe aggressors over the sample in each sex was $2.6 \%$ women $(n=23)$ and $2.8 \%$ men $(n=24)$, not finding statistically significant differences between both sexes $\left(\chi^{2}=0.04, p>.05\right)$.

- In relation to the amount of aggressive behaviour suffered and carried out, the results of the analysis of variance show statistically significant differences according to sex in the indicators of victimization and aggression. Girls have significantly higher mean scores on the level of victimization and boys on the level of aggression. Therefore, girls suffer more aggressive behaviours, and boys perpetrate more behaviours of this type, although the effect size of these differences is small.

- Victims and aggressors of bullying: Differences based on sexual orientation.

- Of the $11 \%$ of severe victims $(n=193)$, the percentage of heterosexuals and non-heterosexuals over the sample in each sexual orientation group was $9 \%$ heterosexual $(n=138)$ and $25.1 \%$ non-heterosexual $(n=55)$, finding significant differences $\left(\chi^{2}=50.48, p<.001\right)$ with a higher percentage of victims in the non-heterosexual group. The percentage of victims in each sexual orientation group, based on the sample in each group, was $37.9 \%$ heterosexual $(n=579)$, $76.9 \%$ gay $(n=10), 33.3 \%$ lesbian $(n=2), 75.8 \%$ bisexual $(n$ $=75$ ) and $60.6 \%$ are not sure of their sexual orientation ( $n$ $=63$ ). Statistically significant differences were found based on sexual orientation $\left(\chi^{2}=78.46, p<.001\right)$, with a higher percentage of gay and bisexual victims.

- Of the $2.7 \%(n=47)$ of the severe aggressors, the percentage of heterosexuals and non-heterosexuals, over the sample in each group of sexual orientation, was $1.7 \%$ heterosexual $(n=38)$ and $0.9 \%$ non-heterosexuals $(n=9)$, finding non significant differences based on sexual orientation $\left(\chi^{2}=\right.$ $0.75, p>.05)$. The percentage of offenders in each sexual orientation group, based on the sample in each group, was $27.4 \%$ heterosexual $(n=419), 38.5 \%$ gay $(n=5), 66.7 \%$ lesbian $(n=2), 45.5 \%$ bisexual $(n=54)$ and $27.9 \%$ participants who are not sure of their sexual orientation $(n=29)$. Statistically significant differences were found based on sexual orientation $\left(\chi^{2}=17.61, p<.05\right)$, with a higher percentage of offenders in the lesbian group.

- The results show that bisexual people have the highest victimization scores. Regarding the level of aggression, significantly higher mean scores are evident in the group of lesbians. Consequently, bisexuals had suffered significantly more aggressive face-to-face bullying behaviours in their lifetime. Finally, lesbians were the ones who had carried out significantly more of this type of behaviour.

These results confirm once again that, although there are antibullying programs and anti-discrimination policies that want to promote respect for sexual diversity from an early age, the legal situation is far from social reality. That is, there are laws that accuse attitudes of rejection and discrimination towards the LGBT community; however, today many people continue to have LGBT-phobic behaviours and attitudes. From early childhood, merely heteronormative messages continue to be received from the media, family, social and social environment and, especially, from the educational community (teachers, audio-visual, educational materials, etc.). Likewise, these results 
place us facing the obligation to continue working on the design of new measures to combat all types of aggression in schools, without forgetting any type of origin that each of them may have (sexual orientation, sexual identity, race, physical, etc.).

Nevertheless, this study has limitations. The biggest limitation has been obtaining the sample, since, on the one hand, bullying is a phenomenon that creates great controversy and some of the randomly selected schools did not agree to participate in this research. On the other hand, although the LGBT community has greater visibility in our society every day, it is very difficult for adolescents to identify themselves as such. Therefore, a low percentage of students who report being non-heterosexual has been obtained. Finally, the use of self-report questionnaires, due to the social desirability bias that they imply.

These results confirm the need to continue developing and implementing bullying prevention and intervention programs. In this regard, we could mention Cyberprogram 2.0 intervention program (Garaigordobil \& Martínez-Valderrey, 2014, 2016, 2018), that has shown a reduction in face-to-face bullying and that, in addition, includes some activities around bullying for orientation reasons sexual. Along these lines, it is necessary to design new activities that promote respect for LGBT people and for those who move away from stereotypes that are marked by heteronormativity. These activities should be aimed at the entire educational community, starting with the youngest students, continuing with the students in adolescent stages, and ending with specific activities for parents and all school workers. It is impossible to achieve a change in the students if the main educators of our society are not educated.

\section{Conflicto de Intereses}

Las autoras de este artículo declaran que no tienen ningún conflicto de intereses.

\section{Referencias}

Abreu, R. L. y Kenny, M. C. (2017). Cyberbullying and LGBTQ youth: A systematic literature review and recommendations for prevention and intervention. Journal of Child \& Adolescent Trauma, 11(1), 81-97. https://doi. org/10.1007/s40653-017-0175-7

Baiocco, R., Pistella, J., Salvati, M., Loverno, I. y Lucidi, F. (2018). Sports as risk environment: homophobia and bullying in a simple of gay and heterosexual men. Journal of Gai \& Lesbian Mental Health, 22(4), 385-411. https://doi.org/10.1080/19359705.2018.1489325

Birkett, M., Espelage, D. L. y Koenig, B. (2009). LGB and questioning students in schools: The moderating effects of homophobic bullying and school climate on negative outcomes. Journal of Youth and Adolescence, 38(7), 989-1000. https://doi.org/10.1007/s10964-008-9389-1

Bogolyubova, O., Skochilov, R. y Smykalo, L. (2015). Childhood victimization experiences of young adults in St. Petersburg, Russia. Journal of Interpersonal Violence, 30(7), 1153-1167. https://oi.org/10.1177/0886260514539849

Camodeca, M., Baiocco, R. y Posa, O. (2018). Homophobic bullying and victimization among adolescents: The role of prejudice, moral disengagement, and sexual orientation. European Journal of Developmental Psychology, 16(5), 503-521. https://doi.org/10.1080/17405629.2018.1466699
COGAM. (2016). Benítez, E. (Ed.), Ciberbullying LGBT-fóbico. Nuevas formas de intolerancia. Grupo de Educación de COGAM. https://www.bienestaryproteccioninfantil.es/imagenes/tablaContenidos03SubSec/4-ciberbullying-lgbt-fc3b3bico-informe-completo-web.pdf

Elipe, P., de la Oliva Muñoz, M. y Del Rey, R. (2017). Homophobic bullying and cyberbullying: Study of a silenced problem. Journal of homosexuality, 65(5), 672-686. https://doi.org/10.1080/00918369.2017.13 33809

Garaigordobil, M. (2013). Cyberbullying. Screening de acoso entre iguales. Screening del acoso escolar presencial (bullying) y tecnológico (cyberbullying). TEA.

Garaigordobil, M. (2015a). Cyberbullying in adolescents and youth in the Basque Country: Prevalence of cybervictims, cyberaggressors, and cyberobservers. Journal of Youth Studies, 18(5): 569-582. https://doi. org/10.1080/13676261.2014.992324

Garaigordobil, M. (2015b). Ciberbullying en adolescentes y jóvenes del País Vasco: cambios con la edad. Anales de Psicologia, 31(3), 1069 1076. https://doi.org/10.6018/analesps.31.3.179151

Garaigordobil, M. (2017). Bullying y cyberbullying: definición, prevalencia, consecuencias y mitos. Fundació per la Universitat Oberta de Catalunya. UOC.

Garaigordobil, M. y Larrain, E. (2017). Cuestionarios Socio-demográficos. Material no publicado.

Garaigordobil, M. y Martínez-Valderrey, V. (2014). Cyberprogram 2.0. Un programa de intervención para prevenir y reducir el ciberbullying. Pirámide.

Garaigordobil, M. y Martínez-Valderrey, V. (2016). Cybereduca cooperativo 2.0. Juego para la prevención del bullying y cyberbullying. http://www.cybereduca.com

Garaigordobil, M. y Martínez-Valderrey, V. (2018). Technological resources to prevent cyberbullying during adolescence: The cyberprogram 2.0 program and the cooperative cybereduca 2.0 videogame. Frontiers in Psychology, 9, 745. https://doi.org/10.3389/fpsyg.2018.00745

Garaigordobil, M. y Oñederra, J. A. (2010). La violencia entre iguales: revisión teórica y estrategias de intervención. Pirámide.

Gegenfurtner, A. y Gebhardt, M. (2017). Sexuality education including lesbian, gay, bisexual, and transgender (LGBT) issues in schools. Educational Research Review, 22, 215-222. https://doi.org/10.1016/j. edurev.2017.10.002

Generelo, J., Garchitorena, M., Montero, P. e Hidalgo, P. (2012). Acoso escolar homofóbico y riesgo de suicidio en adolescentes y jóvenes LGB. COGAM. http://www. felgtb. org/rs/1584/d112d6ad-54ec-438b9358-4483f9e98868/91c/filename.

Martxueta, A. y Etxeberria, J. (2014). Análisis diferencial retrospectivo de las variables de salud mental en lesbianas, gais y bisexuales (LGB) víctimas de bullying homofóbico en la escuela. Revista de Psicopatología y Psicología Clínica, 19(1), 23-35. http://doi.org/10.5944/ rppc.vol.19.num.1.2014.12980

Olweus, D. (1999). Norway. En P. K. Smith, Y. Morita, J. Junger-Tas, D. Olweus, R. Catalano y P. Slee (Eds.), The nature of school bullying. A cross-national perspective (pp. 28-48). Routledge.

Olweus, D. (2013). School bullying: Development and some important challenges. Annual Review of Clinical Psychology, 9, 751-780. https:// doi.org/10.1146/annurev-clinpsy-050212-185516

Orue, I., Calvete, E. y Fernández-González L. (2018). Adaptación de la Escala de Acoso Escolar Homofóbico y magnitud del problema en adolescentes españoles. Behavioral Psychology, 26(3), 437-455.

Pichardo, J. I., De Stéfano, M., Sánchez-Sainz, M., Puche, L., Molinuevo, B. y Moreno, O. (2013). Diversidad sexual y convivencia: una oportunidad educativa. http://www.felgtb.org/rs/3660/d112d6ad-54ec-438b9358-4483f9e98868/234/filename/informe-final-diversidad-y-convivencia.pdf

Toomey, R. B. y Russell, S. T. (2016). The role of sexual orientation in school-based victimization: A meta-analysis. Youth \& Society, 48(2), 176-201. https://doi.org/10.1177/0044118X13483778 